\title{
PREY GROWTH AND SIZE-DEPENDENT PREDATION IN JUVENILE ESTUARINE FISHES: EXPERIMENTAL AND MODEL ANALYSES
}

\author{
J. Kevin Craig, ${ }^{1,5}$ Brian J. Burke, ${ }^{2}$ Larry B. Crowder, ${ }^{3}$ and James A. Rice ${ }^{4}$ \\ ${ }^{1}$ Nicholas School of the Environment and Earth Sciences, Duke University Marine Laboratory, \\ 135 Duke Marine Lab Road, Beaufort, North Carolina 28516-9721 USA \\ ${ }^{2}$ Fish Ecology Division, Northwest Fisheries Science Center, NOAA Fisheries, \\ 2725 Montlake Boulevard East, Seattle, Washington 98112-2097 USA \\ ${ }^{3}$ Nicholas School of the Environment and Earth Sciences, Duke Center for Marine Conservation, \\ 135 Duke Marine Lab Road, Beaufort, North Carolina 28516-9721 USA \\ ${ }^{4}$ Department of Zoology, North Carolina State University, Raleigh, North Carolina 27695-7617 USA
}

\begin{abstract}
The outcome of predation interactions between growing, size-structured predator and prey cohorts is difficult to predict. We manipulated the food resources available to juvenile spot subject to predation from southern flounder in a 60-day replicated pond experiment to test the hypothesis that spot growing slowly would experience higher predation mortality and stronger selection against small individuals than those growing rapidly. A nearly threefold difference in average growth rate between fast- and slow-growth treatments led to twofold higher predation mortality of slow-growing spot. Relative to no-flounder controls, larger spot were overrepresented at the end of the experiment in both treatments, but the magnitude of flounder size selection was much greater in the slow-growth treatment. The experimental results agreed qualitatively, but not quantitatively, with predictions from a prior size-dependent foraging model. In particular, the model significantly underestimated observed shifts in spot size structure to larger sizes. We hypothesized that competitive release and associated increases in spot growth due to thinning by flounder might reconcile this difference, and extended the model to incorporate this process. We then used the model to estimate the relative contribution of these two confounded predator effects (size-selective predation and thinning) to observed shifts in spot size structure. Model simulations indicated that the combined effects of size-selective predation and thinning could account for nearly all of the observed shift in spot size structure, but that thinning was the more important process. Our results highlight the utility of combining experimental and modeling approaches to unravel the complexities underlying interactions between growing, size-structured predator and prey cohorts.
\end{abstract}

Key words: cohort dynamics; competitive release; density-dependent growth; estuarine fishes; flounder; Leiostomus xanthurus; Paralichthys lethostigma; size-dependent predation; size selection; size structure; spot; thinning.

\section{INTRODUCTION}

Interactions between size-structured populations can have profound and often nonintuitive effects on population and community dynamics (Ebenman and Persson 1988). Two pervasive features of natural communities are that ecological performance depends on body size, and individual growth and development are density dependent. A rapidly growing theory emphasizes the interplay between size-dependent and density-dependent ecological processes at the individual level in determining the dynamics of size-structured species at the population and community levels (De Roos et al. 2003).

Predators can alter the densities of prey via consumption and can alter the phenotypes exhibited by

Manuscript received 28 September 2005; revised 26 January 2006; accepted 15 February 2006. Corresponding Editor: A. M. Bronikowski.

${ }^{5}$ E-mail: kevin.craig@duke.edu surviving prey if that consumption is selective. The outcome of predation interactions in aquatic ecosystems is typically size dependent, with the ability of predators to kill and consume prey, or their preferences for particular size prey, dependent on relative body size (Werner and Gilliam 1984, Wilbur 1988). Despite its ubiquity, the consequences of size-dependent predation at the cohort level are not always obvious because both predators and prey are typically size-structured and growing, so that relative body size relationships vary among individuals and are constantly in flux. As a result, both the direction and magnitude of size-selective predation mortality on a size-structured prey cohort can change over ontogeny (Fig. 1; Rice et al. 1997).

Questions have been raised in both the aquatic and terrestrial literature about whether large body size, and by extension fast growth, is always an advantage to prey during interactions with predators (Sogard 1997, Williams 1999). Monotonically increasing benefits of large prey size can be questioned based on optimal foraging 


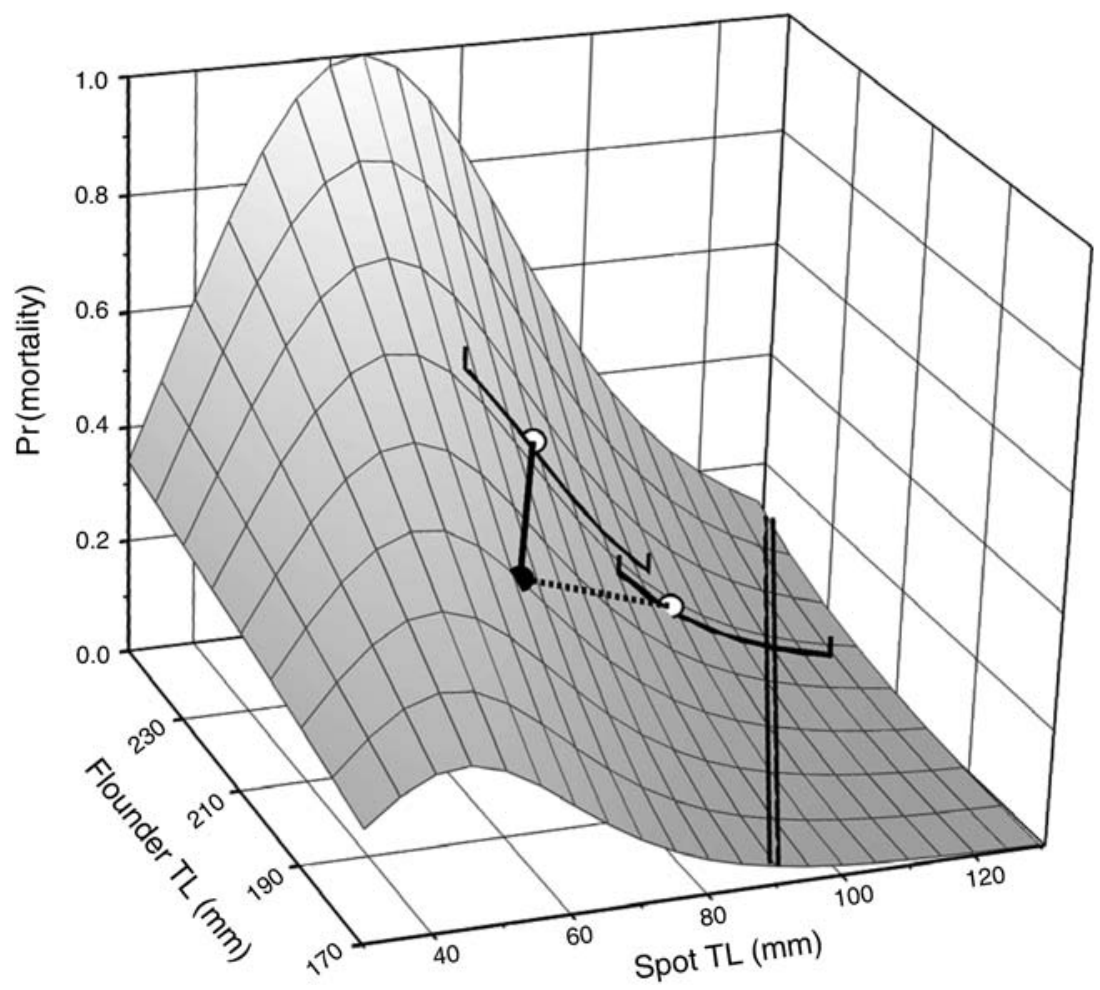

FIG. 1. Response surface describing the daily probability of spot (Leiostomus xanthurus) mortality as a function of southern flounder (Paralichthys lethostigma) and spot total length (TL) from an individual-based, size-dependent foraging model. The model is based on a constant encounter rate and a dome-shaped profitability function (net energy gain per unit handling time weighted by probability of capture) derived from laboratory experiments (see Rice et al. [1997] for details). Note the potential for both positive and negative size selection on either side of the high mortality ridge (flounder TL : spot TL=3.9), depending on the relative sizes and growth rates of spot and flounder. The solid circle indicates the starting mean sizes for both slow- and fast-growth treatments in the pond experiment. Open circles indicate endpoints for the slow-growth (solid line) and fast-growth (dotted line) treatments after 60 days. The two solid lines with vertical bars indicate the 5th and 95th percentiles of the spot length distribution in each growth treatment at the end of the experiment. The double vertical line is the flounder gape limit (flounder TL: spot TL $=1.9$ ).

considerations that suggest larger prey provide greater energetic returns to predators and should be selected (Harper and Blake 1988), or that prey profitability to predators, a potential basis for predator choice, often peaks at intermediate prey sizes (Scharf et al. 2002). In addition, recent studies have identified costs associated with rapid growth that may increase vulnerability to predators (Munch and Conover 2003, Stoks et al. 2005) or have negative consequences for other aspects of fitness (Arendt 1997, Metcalfe and Monaghan 2001). In a review of herbivorous insects, a nearly equal number of studies rejected as supported the hypothesis that rapid herbivore growth leads to lower mortality from predators and parasitoids (Williams 1999). The benefits of rapid prey growth can also depend on predator growth, as growing predators may "surf" the changing size distribution of their growing prey such that prey remain within a relative size window where they are vulnerable to predation (Pope et al. 1994). Recent theoretical models have emphasized the importance of this predation window in driving a variety of alternative population and community dynamic behaviors (Claessen et al. 2002, Persson et al. 2003). Despite the pervasiveness of size-dependent predation, relatively few studies have investigated interactions between growing, size-structured predator and prey cohorts, particularly using an experimental approach.

In addition to molding the phenotypic distribution of prey through selective consumption, predators can also alter prey phenotypes via indirect mechanisms (Peacor and Werner 2000, Relyea 2002). For example, predators can alter prey size structure through both positive and negative effects on prey growth rates. Predators can negatively impact prey growth rates through intimidation, such that prey decrease foraging activity in the presence of predators, or shift to habitats with lower availability or quality of food resources (Preisser et al. 2005). Predators can positively impact prey growth rates by thinning prey cohorts and inducing competitive release among survivors (Wilbur 1988), or otherwise altering the per capita availability of prey food resources (Peacor 2002). These positive and negative effects of predators on prey growth can occur simultaneously and trade off against one another, making it difficult to identify mechanisms underlying predator effects on prey phenotypes. Even so, growing evidence indicates that 
predator-induced changes in the phenotypes of surviving prey can have important and widespread consequences for community dynamics (Werner and Peacor 2003, Preisser et al. 2005).

While predators can impact the size structure of prey directly by selective consumption and indirectly by thinning and associated competitive release of survivors, these two processes have rarely been considered in concert. For gape-limited predators that selectively consume small prey, predation not only removes smaller individuals from the prey cohort, but also simultaneously decreases the density of prey, potentially leading to increased growth of survivors released from competition. In this case, these two distinct processes (i.e., sizeselective predation and thinning) alter prey size structure in the same direction (shift to larger sizes) and, because they occur simultaneously, are confounded in many studies of predation. For predators that selectively consume large prey, the simultaneous influence of sizeselective predation and thinning have opposite effects, with size-selective predation shifting prey size structure to smaller sizes (due to consumption of larger individuals) and thinning shifting prey size structure to larger sizes (due to increased growth of survivors). In this case, no effect of the predator on prey size structure may be apparent when, in fact, two opposing effects occur. Hence, separating the relative contributions of sizeselective predation and thinning to shifts in prey size structure is particularly problematic because the two processes cannot occur independently (a single act of consumption simultaneously alters both the size distribution and the density of survivors) and the magnitude and direction of the two processes can vary through time as predators and prey grow (Fig. 1).

We conducted a replicated pond experiment and modeled the interaction between juvenile spot (Leiostomus xanthurus) and southern flounder (Paralichthys lethostigma) to investigate how prey growth dynamics impact prey mortality and size structure during interactions with a growing predator. We compared our experimental results with predictions from a previously developed individual-based foraging model based on flounder foraging gains and costs as a function of relative body size (Fig. 1; Rice et al. 1997). While the model performed reasonably well in predicting the effects of flounder predation on spot mortality, it could not fully account for observed shifts in spot size structure. We hypothesized that flounder altered spot size structure not only via size-selective predation, but also by thinning spot cohorts and thereby altering the growth rates of survivors released from intraspecific competition. To test this hypothesis we extended our model to incorporate density-dependent growth of surviving spot as the cohort was thinned due to flounder predation (J. K. Craig, J. A. Rice, L. B. Crowder, and D. A. Nadeau, unpublished manuscript). We then conducted simulation experiments with the model to determine the relative importance of these confounded predator effects (i.e., size-selective predation and thinning) in generating the final size distribution of spot observed in the experiment.

\section{Methods \\ Predictions}

We tested the hypothesis that spot cohorts with initially similar size distributions but growing at different rates would experience different amounts of predation mortality and selection on size structure. First, we predicted that if spot initially of a size that would be highly vulnerable to flounder (i.e., near the peak of the flounder profitability function, Fig. 1) were provided with additional food resources, they would grow more rapidly, experience a more rapid decline in vulnerability to flounder, and, as a result, incur lower predation mortality compared to spot with more limited food resources. Second, we predicted that both slowand fast-growing spot cohorts would experience directional selection against smaller individuals as they grew toward the flounder gape limit, shifting both size distributions to larger sizes. However, because slow growth increases the duration that spot are vulnerable to flounder (and the cumulative probability of predation mortality), we predicted that the magnitude of this selection against smaller individuals would be greater for slow-growing spot.

\section{Pond experiment}

To test the above predictions we conducted a replicated, 60-day pond experiment in two shallow, mud-bottom ponds (420- $\mathrm{m}^{2}$ each), each divided into six $70-\mathrm{m}^{2}$ pie-wedge sections (see Appendix A for details). The experimental design consisted of three replicates of each of two treatments: fast-growing spot with flounder and slow-growing spot with flounder. To separate the effects of flounder predation on spot mortality and size structure from those due to spot growth, we also established three replicates of each of two controls: fast-growing spot without flounder and slow-growing spot without flounder. By comparing spot mortality and size structure between growth rate treatments with flounder relative to those without, we could isolate the effects of spot growth on mortality and size structure that were the result of flounder predation. An assumption of our approach is that there are no interactive effects of flounder presence vs. absence and spot growth rate on spot mortality and size structure (see Discussion, Appendix A). We allocated the treatments randomly among the 12 pond sections with the constraint that all combinations of flounder and no-flounder, and fastgrowing and slow-growing spot replicates were represented in each pond (though not necessarily in equal numbers of replicates).

Juvenile spot and juvenile southern flounder were collected from local waters and maintained in flowthrough tanks. After measuring a subsample (mean $\pm \mathrm{SD}$, total length [TL], $65.8 \pm 6.9 \mathrm{~mm}$; range, $35-87 \mathrm{~mm} ; n=$ 
324 spot), 200 spot were stocked into each of the 12 pond replicates (2400 spot total) followed by random assignment of three flounder to each of six pond replicates. Each flounder was measured and an individual visual implant tag inserted prior to stocking (TL, 198.5 \pm 6.6 $\mathrm{mm}$; range, 189-210 mm; Appendix B). Initial flounder lengths were about threefold those of spot (Fig. 1, range of means among replicates, flounder TL: spot TL, 3.023.07 ), and all spot were initially within the flounder gape limit (range of individual length ratios within replicates, 5.67 to 2.28 ; gape limit $=1.9$ ). Hence, spot were highly vulnerable to flounder predation at the beginning of the experiment. Sinking fish pellets larger than the mesh separating the sections ( 8 vs. $5 \mathrm{~mm}$ diameter) were provided at two different rates to generate the fast- and slow-growth treatments. Spot feed readily on pellets and uneaten pellets increase benthic invertebrate production that is commonly consumed by spot as well (Hodson et al. 1981, Crowder et al. 1994), while flounder prefer live, mobile prey. We fed spot at $\sim 26 \%$ body mass per day in the fast-growth treatment and $\sim 2 \%$ body mass per day in the slow-growth treatment. On day 60 the ponds were drained and all surviving spot and flounder were recovered and measured.

\section{Analysis}

Spot mortality due to flounder predation and unknown sources was estimated from an exponential mortality model (Cushing 1981, Crowder 1994):

$$
N=I\left(1-D_{\mathrm{c}}-D_{\mathrm{f}}\right)^{T}
$$

where $N$ is total number of spot recovered per replicate, $I$ is initial number of spot stocked per replicate, $D_{\mathrm{c}}$ is mean daily per capita probability of dying due to factors other than flounder predation (i.e., background mortality), $D_{\mathrm{f}}$ is mean daily per capita probability of dying due to flounder predation, and $T$ is duration of the experiment (60 days). $D_{\text {c }}$ was estimated using spot survival data from pond replicates without flounder and setting $D_{\mathrm{f}}=0 . D_{\mathrm{c}}$ was then used with spot survival data from the replicates with flounder to solve for $D_{\mathrm{f}}$ (Appendix A). This approach assumes that the presence of flounder had no effect on the daily per capita probability of spot dying due to other factors (i.e., background and flounder predation mortality act independently). Individual flounder growth rates were calculated as the difference between initial and final length divided by the experiment duration. Average spot growth rates were calculated as the difference between initial and final median lengths in each replicate divided by the experiment duration.

We used a $t$ test on arcsine square-root transformed proportions to compare mortality between fast- and slow-growth treatments. We used the nonparametric Watson goodness-of-fit test (Watson 1962), based on the integrated difference between two cumulative distribution functions (CDFs), to compare spot length distributions between each growth rate treatment and its respective control (e.g., slow-growing spot with flounder vs. slow-growing spot without flounder).

\section{Model simulations}

We compared our experimental results with predictions from a previously developed individual-based foraging model (Rice et al. 1997). We used the estimates of $D_{\text {c }}$ (Eq. 1) averaged separately over fast- and slowgrowth replicates in the no-flounder controls to simulate spot background mortality for the two growth treatments in the presence of flounder. To simulate spot growth rates, we first fit separate lognormal distributions of growth rates that best matched the final length distribution of each no-flounder replicate by systematically searching over all combinations of a range of medians $(0.1-2.0 \mathrm{~mm} / \mathrm{d} ; 0.1$ increments) and standard deviations $(0.05-20 \mathrm{~mm} / \mathrm{d} ; 0.01$ increments) in spot growth. We assumed a lognormal distribution because final spot lengths were positively skewed relative to initial lengths. We averaged the results of 30 model runs with different random number seeds and chose the parameters of the lognormal distribution that minimized the sum of the squared differences between the length distribution predicted by the model and that observed in each noflounder control replicate at the end of the experiment. These parameter values were then averaged over replicates within fast- and slow-growth treatments and used in further simulations that included flounder predation.

We simulated flounder predation as the product of the daily probability that an individual spot encountered a flounder and the probability that the encountered flounder attacked and captured the spot (Fig. 1, Rice et al. 1997). In a prior model application to a similar pond experiment, Rice et al. (1993) estimated the daily probability that an individual spot encountered a flounder by back-calculating that needed to yield the observed mortality, assuming that the probability of attack and capture followed a dome-shaped profitability function derived from laboratory experiments. We assumed this previously estimated encounter probability after scaling for the difference in spot (2.86 vs. 5.0 individuals $/ \mathrm{m}^{2}$ ) and flounder (0.04 vs. 0.08 individuals/ $\mathrm{m}^{2}$ ) densities between the current and previous experiment (Gerritsen and Strickler 1977; encounter probability $=0.02$ flounder $/$ spot $/ \mathrm{d}$ ). While the daily probability that an individual spot encountered a flounder was constant over the simulation (because the number of flounder did not change), the encounter rate of flounder with spot (total number of encounters per day) decreased over time as spot abundance declined. The particular flounder encountered was chosen at random from the three available in the simulated replicate. Once an encounter occurred, the probability that the spot was attacked and captured was determined from the relative profitability of the spot to the encountered flounder based on the flounder: spot size ratio (Fig. 1, Rice et al. 1997). Individual flounder grew 

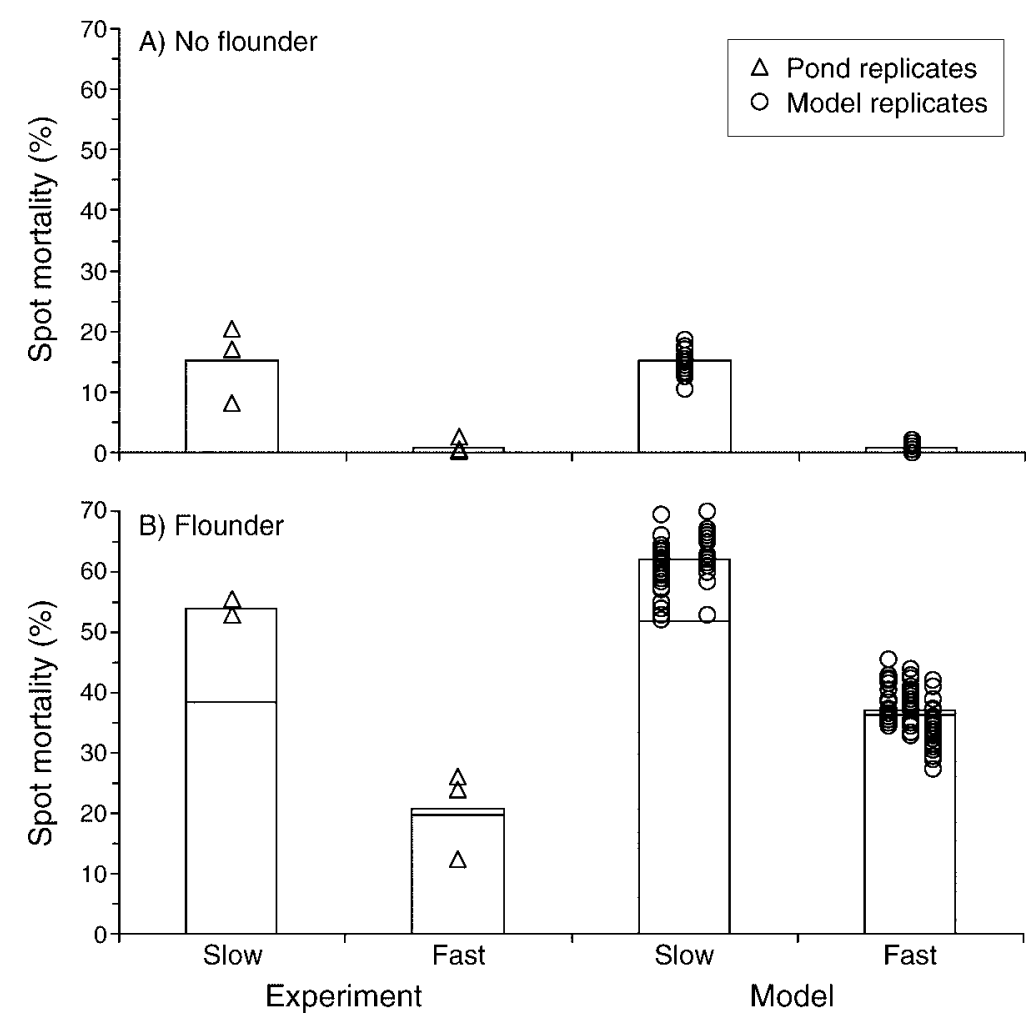

FIG. 2. Observed and model-simulated mortality of slow- and fast-growing spot in the (A) absence and (B) presence of flounder. Horizontal lines in (B) divide mean total mortality into background (upper) and flounder predation (lower) components. Total spot mortality in individual pond replicates is indicated by triangles. Circles indicate spot mortality in 30 model simulations of each pond replicate (adjacent sets of circles). One slow-growth replicate was omitted because only two of three flounder were recovered.

over the simulation according to their daily individual growth rate observed in the ponds.

\section{RESULTS}

Spot grew fast compared to flounder in the pond experiment, with relative size of spot in both growth treatments (flounder: spot TL ratio) moving down the descending slope of the vulnerability function toward the flounder gape limit (Fig. 1, Appendix A). Average length ratios shifted from $\sim 3.1$ at the beginning of the experiment to 1.92-2.03 among replicates of the fastgrowth treatment (1.39-2.64 among individual survivors) and 2.34-2.57 among replicates of the slowgrowth treatment (1.59-3.06 among individual survivors) by the end of the experiment. Flounder growth varied widely both within and among replicates $(0.00-0.75 \mathrm{~mm} / \mathrm{d}$, Appendix B), but did not differ between slow- and fast-growth treatments (mean \pm SD; slow growth, $0.39 \pm 0.289 \mathrm{~mm} / \mathrm{d}$; fast growth, $0.31 \pm$ $\left.0.248 \mathrm{~mm} / \mathrm{d} ; F_{1,4}=0.21, P=0.68\right)$. Given these growth rates and the spot and flounder sizes remaining after 60 days, nearly all spot surviving to the end of the experiment were still vulnerable to flounder in the slow growth treatment (98\%) while $\sim 39 \%$ of spot in the fastgrowth treatment had surpassed the flounders' gape limit (Fig. 1). Assuming constant growth rates of both spot and flounder over the experiment, we estimated that all spot in the fast-growth treatment remained vulnerable to flounder until about day 30 , after which some spot became invulnerable.

\section{Spot mortality}

Spot mortality after 60 days in the ponds was low in the absence of flounder but differed between growth treatments, averaging $15.2 \%$ for spot in the slow-growth treatment and $0.8 \%$ for spot in the fast-growth treatment (Fig. 2A, $t_{4}=4.46, P=0.011$; Appendix A). Total spot mortality in the presence of flounder was $\sim 54 \%$ in the slow-growth treatment and $21 \%$ in the fast-growth treatment, a 2.6-fold difference (Fig. 2B, $t_{4}=5.99, P=$ $0.004)$. When calculated relative to background mortality in the appropriate no-flounder control, mortality of slow-growing spot attributable to flounder predation was nearly twofold that of fast-growing spot (38.5\% vs. $19.8 \% ; t_{4}=2.88, P=0.045$ ).

In model simulations of the no-flounder controls, mortality due to unknown sources (i.e., background mortality) was nearly identical to that observed in the ponds (Fig. 2A). In model simulations with flounder predation, total predicted mortality of spot was within 

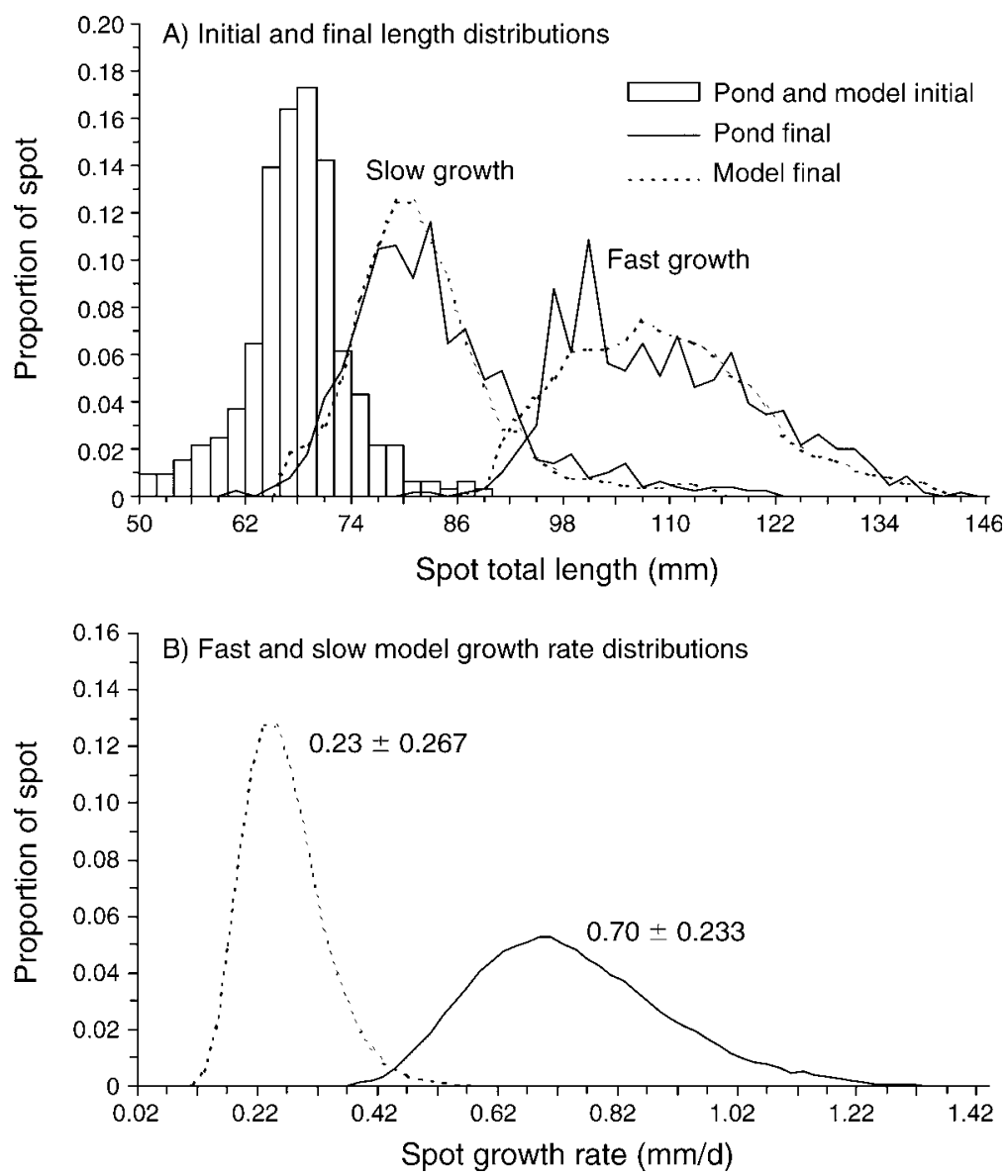

FIG. 3. (A) Observed and model-simulated length distributions of slow- and fast-growing spot in the no-flounder controls of the pond experiment. Histogram bars represent initial distributions for the pond and model. (B) Lognormal growth rate distributions (median $\pm \mathrm{SD}$ ) that provided the best fit to the pond experiment.

$8-16 \%$ of that observed in the experiment, averaging $62.1 \%$ in the slow-growth treatment and $37 \%$ in the fastgrowth treatment, a 1.7-fold difference (Fig. 2B). Mortality attributable to flounder predation was 1.4fold higher in simulations of slow-growing spot compared to fast-growing spot. The model, however, overestimated spot mortality for both growth treatments, and this difference was greater for fast- than for slowgrowing spot. For example, relative to that observed in the experiment, the model predicted $34.5 \%$ higher mortality of slow-growing spot but $75 \%$ higher mortality of fast-growing spot. This asymmetry in the difference in mortality predicted by the model and that observed in the experiment suggests the higher mortality in the model was not solely a result of scalar differences (perhaps attributable to our assumption of a constant encounter probability).

\section{Spot size structure}

The final length distributions of slow- and fastgrowing spot in the no-flounder controls differed considerably from each other and from the initial spot length distribution (Fig. 3A, Appendix A). There was little overlap in the final lengths of fast- and slow-growing spot in the no-flounder controls $\left(8.7 \%, U_{30,30}^{2}=0.23, P<\right.$ 0.001 ), or between each final length distribution and the initial lengths stocked in the ponds (slow growth, 2.9$28.0 \%, U_{26,30}^{2}=0.688, P<0.001$; fast growth, $0-0.51 \%$, $\left.U_{26.30}^{2}=2.05, P<0.001\right)$, indicating that the different feeding rates generated strong differences in spot growth. The best-fit growth rate distribution in the model simulations without flounder resulted in final spot lengths that agreed well with those observed in the noflounder controls (Fig. 3A), suggesting that a lognormal distribution is a good representation of spot growth. The median growth rate that provided the best fit to the final length distributions was about threefold higher for fastgrowing than for slow-growing spot (Fig. 3B).

The presence of flounder shifted the final spot length distribution in the ponds to larger sizes relative to the controls, and the magnitude of this effect was much greater for slow- than for fast-growing spot (Fig. 4A, B, Appendix A). This was particularly evident in the final spot CDFs from the experiment, in which the presence of flounder resulted in proportionally fewer fish in the smaller length classes for both growth treatments (Fig. 

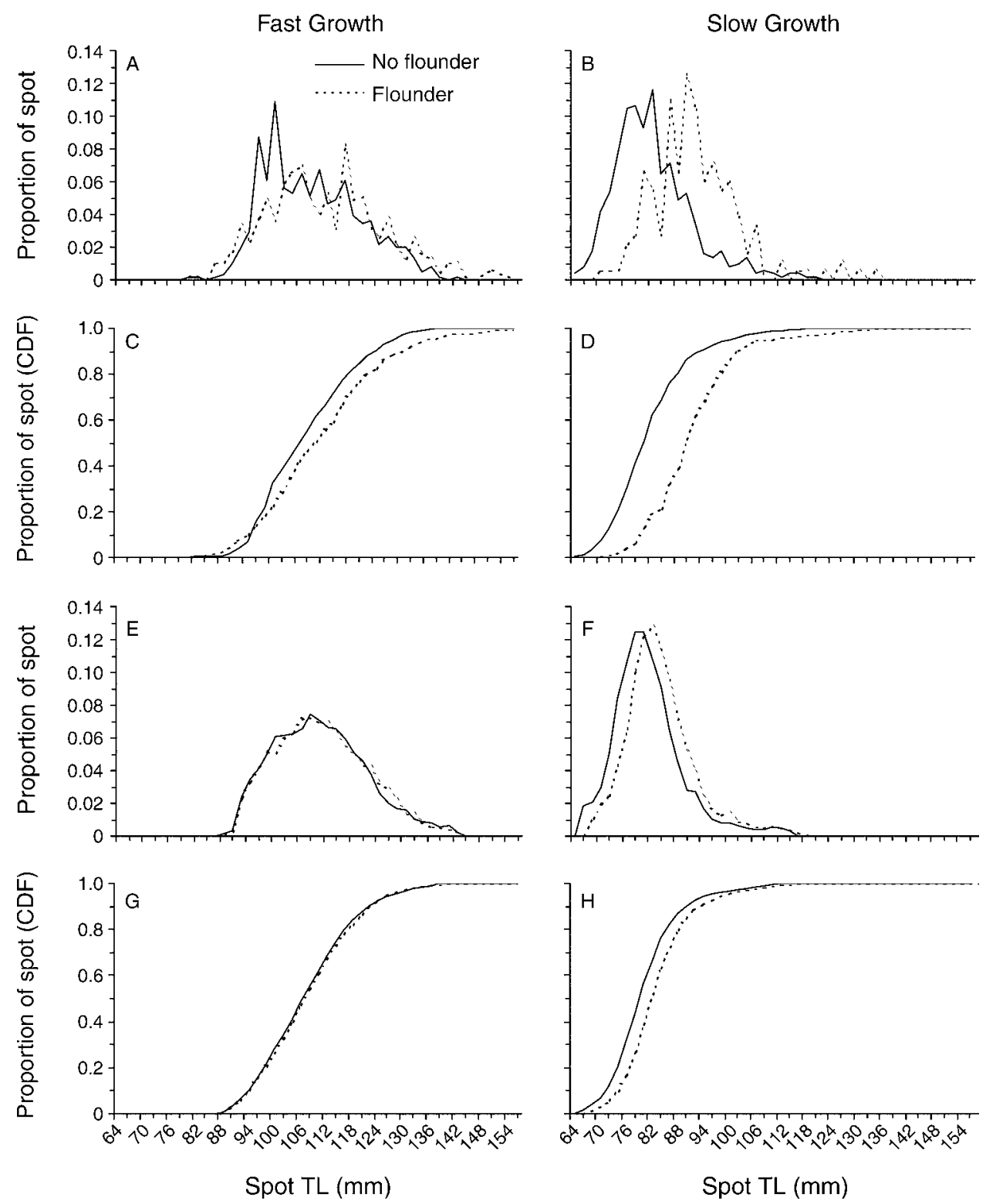

FIG. 4. Final length distributions and cumulative distribution functions (CDF) of spot in the experiment (A-D) and in model simulations $(\mathrm{E}-\mathrm{H})$. Left-hand panels are for the fast-growth treatment, and right-hand panels are for the slow-growth treatment. Distributions are the average over the replicates weighted by the number of survivors.

4C, D). The magnitude of the shift to larger spot sizes (i.e., the area between the respective flounder and noflounder CDF), however, was $\sim 2$.6-fold greater for slow- than for fast-growing spot. This difference in size structure in the presence vs. absence of flounder was significant for the slow-growth treatment $\left(U_{26,30}^{2}=0.266\right.$, $P<0.01)$ but not the fast-growth treatment $\left(U_{39,30}^{2}=\right.$ $0.028, P>0.5)$.

In model simulations, the final length distributions of spot subject to flounder predation also shifted to larger sizes, but the overall magnitude of this effect was much smaller than that observed in the experiment (Fig. 4E$\mathrm{H})$. In fact, the final CDF of fast-growing spot in the model was nearly indistinguishable from the no-flounder control (Fig. 4G, $U_{29,29}^{2}=0.0007, P>0.5$ ). In contrast, the shift in length distribution of slow-growing spot was significantly different from the control in the model $\left(U_{25,44}^{2}=0.213, P=0.034\right)$, but the shift was only about one-third of that observed in the experiment (compare Fig. 4D, H). The weaker selection on spot size distributions in the model simulations compared to the experiment, despite the higher predation mortality incurred by spot in the model, suggests some other process not included in the model impacted spot size structure in the experiment. 
One possibility for the discrepancy in the magnitude of flounder effects on spot size structure between the model and experiment is that surviving spot in the experiment were released from competition and grew faster as the cohort was thinned by flounder predation, further shifting spot size distributions to larger sizes, a process not included in the model. To investigate this possibility, we extended the model to incorporate a functional relationship between spot growth rate and spot density developed from a previous density-dependent growth experiment with spot in the same ponds $(\mathrm{J}$. K. Craig, J. A. Rice, L. B. Crowder, and D. A. Nadeau, unpublished manuscript; Appendix C). This previous experiment revealed a nonlinear decline in spot growth rate across a fivefold increase in spot density due to competition for benthic food resources; spot densities in the current experiment were fully bracketed by the values tested by J. K. Craig, J. A. Rice, L. B. Crowder, and D. A. Nadeau (unpublished manuscript), and were, therefore, high enough to show density dependence. To determine if density-dependent growth associated with thinning of the spot cohort could reconcile the experimental and model results, as well as to investigate the relative importance of size-dependent predation and thinning to the observed shift in spot size structure, we conducted simulation experiments of the slow-growth treatment with: (1) the combined effects of size-dependent predation and density-dependent growth due to thinning, (2) size-dependent predation only (no competitive release and increased growth of survivors), and (3) thinning only (with equivalent mortality to that observed in the experiment but random with respect to size). By comparing shifts in spot size structure predicted by the model incorporating either or both of these processes to that observed in the experiment, we could infer the relative importance of size-dependent predation and thinning in generating the observed shift in spot sizes (Appendix D).

When both size-dependent predation and thinning were simulated, the model accounted for $91 \%$ of the shift to larger spot sizes observed in the experiment (Fig. 5A). Size-dependent predation alone could only account for $18 \%$ of the observed shift, and the final model CDF did not overlap with that observed in the pond replicates with flounder (Fig. 5B). When simulated mortality was random rather than size dependent but spot growth rates were density dependent (i.e., increasing over time as the cohort was thinned), the shift in spot size structure was $\sim 88 \%$ of that observed, and the final model length distribution overlapped considerably with that observed in the ponds (Fig. 5C).

\section{Discussion}

While a variety of fitness benefits undoubtedly attend large body size, the generality that large size is always beneficial to prey during interactions with a predator has been questioned (Sogard 1997, Williams 1999). This is because achieving large body size typically requires rapid growth, which has potential costs that may increase vulnerability to predators (Munch and Conover 2003), and because both the magnitude and direction of size-selective predation mortality can vary across the range of predator and prey body sizes (Rice et al. 1997). We found that a 2.8-fold difference in average growth rate between fast- and slow-growing spot cohorts led to twofold higher predation mortality of slow-growing spot, indicating that rapid growth was highly beneficial to prey under the experimental conditions we investigated. Our model simulations indicate this difference in mortality derived from differences in size-dependent foraging success by flounder that developed over time as spot cohorts with initially similar size distributions grew at different rates. In aquatic ecosystems, the benefits of rapid growth have typically been inferred from sizeselection studies that do not account for the costs of growth or the changes in vulnerability to predators that occur with changing size relationships between growing predators and prey (Sogard 1997). Our results provide strong empirical support for the benefits of rapid growth of a size-structured prey cohort during interactions with a growing predator under more dynamic experimental conditions than have previously been investigated.

The outcome of our experiment may have been dramatically altered, however, if the relative sizes and growth rates of spot and flounder had been different (Rice et al. 1997). While our model predicts that rapidly growing prey that remain to the right of the highmortality ridge (near the flounder gape limit) will experience lower mortality, it also predicts that rapid growth of prey to the left of this ridge (where profitability has not yet reached a maximum) will experience higher mortality (Fig. 1). This predicted negative effect of rapid growth is based on the assumption that flounder selectively consume larger, more profitable spot, and could have occurred, for example, if spot cohorts were initially smaller in size or if flounder had grown more rapidly, such that larger, faster-growing spot became more profitable over time (Crowder et al. 1994). Flounder growth was relatively slow in our experiment, however, such that vulnerability to predation declined over time as both fast- and slowgrowing spot cohorts approached the flounder gape limit (though at different rates). Because prey often have faster relative growth rates than predators, the interplay between initial predator and prey sizes and growth rates in determining the duration that prey are vulnerable prior to survivors reaching a size refuge is likely important in many natural systems (Olson 1996, Persson et al. 1996). Prey vulnerability functions typically peak at intermediate prey sizes, however, suggesting that the effects of a given prey growth rate on prey mortality may differ qualitatively depending on other prey characteristics (i.e., the distribution of prey body sizes) as well as external conditions (i.e., predator sizes and growth rates). 


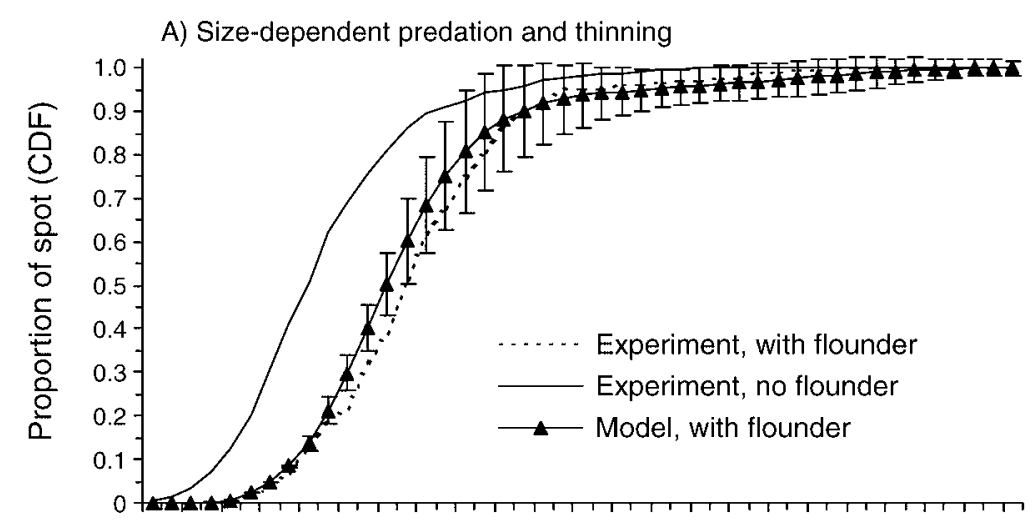

B) Size-dependent predation only
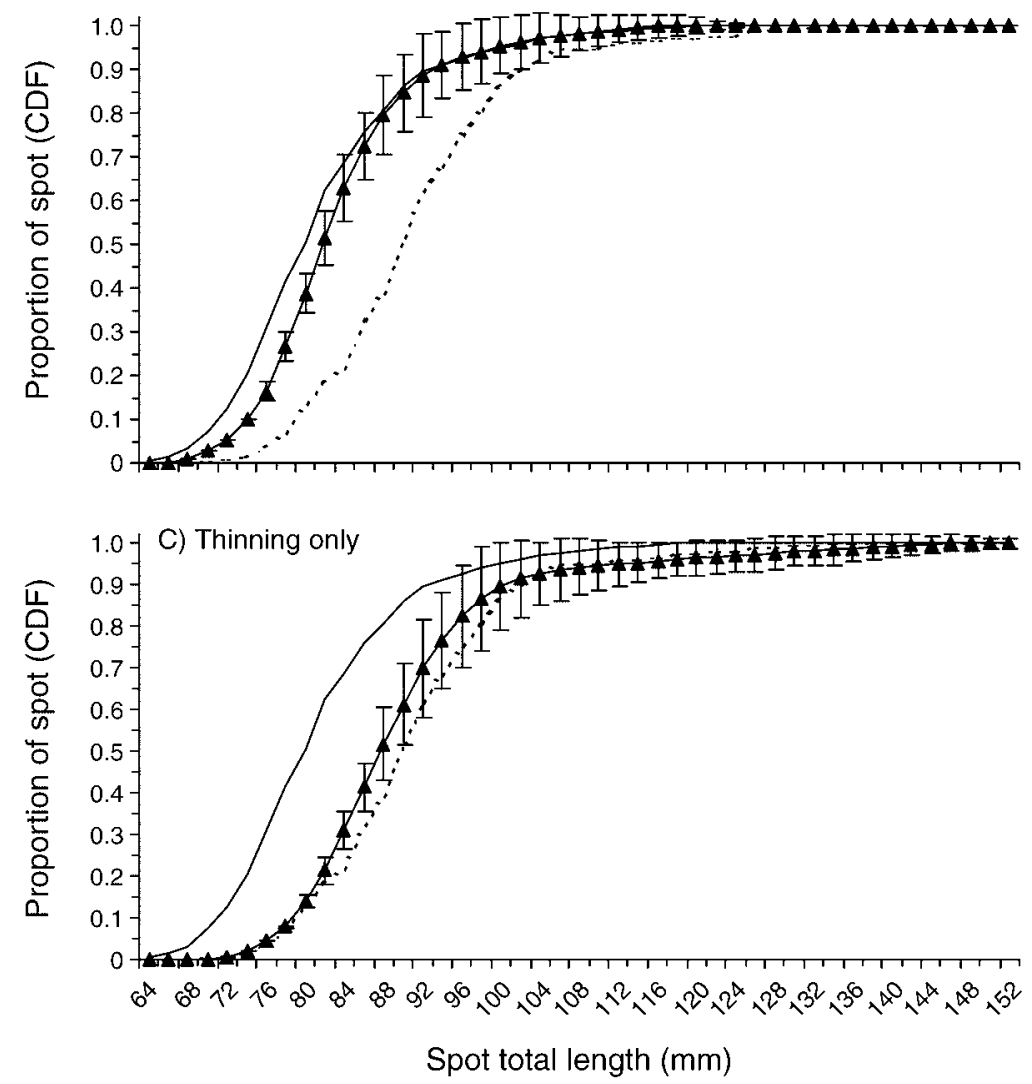

FIG. 5. Combined and separate contributions of size-dependent predation and thinning to observed shifts in spot size structure in model simulations of the slow-growth treatment. Lines without symbols and error bars are the final CDFs from the experiment in the presence (dotted lines) and in the absence (solid lines) of flounder. Lines with triangles and error bars ( \pm 2 SD, 30 replicate simulations) are model simulations of slow-growing spot subject to flounder predation.

Differences in mortality between fast and slow growing spot in the pond experiment agreed qualitatively, but not quantitatively, with predictions from a previously developed individual-based foraging model (Rice et al. 1997). The higher mortality predicted by the model compared to that observed for both growth rate treatments in the experiment was in part due to overestimation of the probability that spot encounter flounder. This is not surprising given the inherent variability in encounter rates between highly mobile predators and prey (Sih and Christensen 2001), and our assumption of a constant daily encounter probability based on a prior experiment (Rice et al. 1993). This assumption, however, can only account for proportional differences in mortality between the model and the experiment, while spot that were growing rapidly had much lower observed than predicted mortality compared to spot that were growing slowly. Size-based processes in our model are driven solely by variation in spot profitability to flounder (i.e., net energy gain per 
unit handling time, weighted by probability of capture) and, for the size ranges considered here, profitability is driven largely by size-dependent capture success. This suggests that larger, faster-growing spot were better able to evade flounder in the ponds than our model would suggest, and is consistent with evidence that laboratory experiments used to parameterize this and similar models tend to overestimate predator capture success and underestimate prey escape responses relative to more open systems (Lima 1998). Size-dependent encounter probabilities (increasing with spot size) are not a plausible explanation for the differences in mortality we observed between the growth treatments, as model simulations (not shown) suggest the offsetting effects of body size on probability of encounter and probability of attack and capture result in little to no difference in predation mortality between fast- and slow-growing spot cohorts. Our results suggest that simple, size-based foraging models such as ours may capture the qualitative dynamics of interactions between growing predators and prey, but may lack the sophistication to make quantitative predictions, particularly for predators and prey that exhibit complex and temporally varying size relationships.

The relative importance of multiple predator effects on prey phenotypes and their consequences for population and community dynamics is receiving increased attention (Relyea 2002, Werner and Peacor 2003). Despite the fact that size-dependent predation and density-dependent growth are fairly ubiquitous in aquatic communities, studies that address their relative contribution to the size structure of a prey cohort are rare. Somewhat surprisingly, thinning and associated increased growth of survivors appeared more important than selective consumption of small spot in explaining the shift to larger spot sizes in the presence of flounder. A previous experiment at spot densities encompassing those reported here and within the range of reported field densities revealed that growth rates declined exponentially with increasing spot density (J. K. Craig, J. A. Rice, L. B. Crowder, and D. A. Nadeau, unpublished manuscript), indicating that density-dependent growth, perhaps in association with predator thinning, is likely important in natural systems as well. The larger contribution of thinning is consistent with studies in some invertebrate and anuran communities indicating that the positive effects of thinning on surviving prey growth rates can be large relative to other (indirect) predator effects, such as predatorinduced decreases in prey foraging activity (Van Buskirk and Yurewicz 1998, Brodin and Johansson 2002). Our approach was not designed to assess similar potential indirect effects of flounder because prior results suggested they were not important (Crowder et al. 1994). Also, our model slightly underestimated the shift in spot size structure to larger sizes observed in the pond experiment (Fig. 5), while we would have expected the model to overestimate the observed shift if flounder had strong, negative indirect effects on spot growth rates in the ponds. The effect of thinning on spot size structure was not apparent in previous investigations of sizedependent predation between spot and flounder because either spot densities were maintained by replenishing those consumed by flounder (Crowder et al. 1994) or spot did not grow due to low food availability (Rice et al. 1993). While size-dependent predation had important effects in these earlier experiments, under the circumstances we investigated here, it did not appear very important in shaping the size structure of spot. This emphasizes the need to consider multiple predator effects across a broad range of predator and prey phenotypes, densities, and resource levels to fully understand how predators shape the phenotypic distributions of prey.

Understanding the relative importance of size-dependent predation and thinning on prey size structure is particularly problematic because, unlike other effects of predators, they cannot occur independently of each other. A single act of size-selective consumption simultaneously alters both the density and the size distribution of survivors. For this reason we suspect that these two processes are confounded in many studies of predation. The only other study addressing the relative importance of size-selective predation and thinning on prey phenotypes also reported that thinning had much more important effects on growth and body size than did selection (Relyea 2002). The effects of selection may be difficult to detect in experimental situations, however, if selective mortality results in a compensatory phenotypic shift in survivors (Relyea 2002), or if the direction of selection changes over the course of an experiment (Crowder et al. 1994). While size-selective predation and thinning had the same directional effects on spot size structure in our experiment (shift to larger spot sizes), we would predict opposing effects for alternative sizes where flounder are selective for larger spot (Fig. 1). For example, when spot are small relative to flounder, we would predict that selective consumption of large spot, which would shift the size distribution to smaller sizes, would be masked by competitive release and increased growth of survivors, which would shift the size distribution to larger sizes. Hence, the effects of thinning and size-selective predation may act in the same direction or in opposite directions, and the direction and relative magnitude of these effects on the distribution of prey sizes may change continuously as predators and prey grow. Failure to consider variation in the relative magnitude of the full suite of mechanisms by which predators can impact prey phenotypes may contribute to relatively high variability in the perceived magnitude of predator effects among systems (Relyea 2002). However, the potential for multiple predator effects to act simultaneously and in the same, or in opposite, directions over alternative ranges of the same predator and prey phenotypes within a system, as suggested by our results, has not been previously 
considered. This suggests that we must not only consider multiple predator effects, but also how the magnitude and direction of these effects change over the ontogeny of the interaction, in order to understand the net effect of predators on prey phenotypes.

The relatively small contribution of size-dependent predation in explaining the observed shift in spot size structure may seem at odds with the relatively large differences in spot mortality between the fast- and slowgrowth treatments. Relative body size influences vulnerability to predators first, by determining the proportion of individuals in the cohort that are susceptible to predation (i.e., within the predator gape limit), and second, by determining size-based variation in vulnerability among prey that are susceptible. Our results indicate the probability of flounder predation mortality declined over the experiment for both growth rate treatments as spot increased in size relative to flounder, but only in the fast-growth treatment did a significant number of spot surpass the flounder gape limit and reach an invulnerable size (Fig. 1). This suggests the difference in mortality between slow- and fast-growing spot that we observed in the experiment may have been more related to the proportion of spot susceptible to flounder, rather than size-based variation in vulnerability among this subset of the spot cohort. The generally low predation mortality in the experiment and the fact that some spot grew to invulnerable sizes suggests sizedependent predation may have only been important for a portion of the experiment and not for all individuals, potentially contributing to the greater relative influence of thinning on spot size structure. Under alternative conditions where predation is more intense (e.g., higher predator densities) and acts over a longer period, the relative magnitude of these two processes may differ. At least one study has reported that size-dependent mortality had a larger effect on size structure than did densitydependent growth (though not necessarily associated with thinning; Sinclair et al. 2002). A fuller understanding of factors driving the within-season dynamics of size-dependent interactions between growing predators and prey is needed, particularly as recent theoretical models suggest that variation in these within-season dynamics can have large impacts on prey population structure and dynamics over longer temporal scales (De Roos et al. 2003).

The role of predators in aquatic communities is complex, involving both lethal and nonlethal effects on prey populations that can propagate to other species in the community and potentially feedback on predator population dynamics (De Roos et al. 2003, Werner and Peacor 2003). We have shown that the direct effect of size-selective predation and the indirect feedback on prey growth due to thinning can have important and sometimes hidden effects on prey survival and the phenotypes of survivors. The interplay between these two processes is dynamic with the relative importance of each likely varying over the ontogeny of the interaction between growing predator and prey cohorts. Unraveling these complex interactions is important given that both size-dependent and density-dependent processes appear to be key factors in understanding population and community dynamics in size-structured populations.

\section{ACKNOWLEDGMENTS}

We thank the University of North Carolina-Chapel Hill Institute of Marine Sciences for access to the pond facilities. We thank G. Bath, M. Crawford, L. Eby, C. McClellan, C. Seegers, and L. Shewmon for assistance with the experiment, K. Rose for his contribution to initial model development, and J. Buckel, C. Greene, P. Levin, S. Searcy, and two anonymous reviewers for comments that improved the manuscript. This research was supported by the National Sea Grant College Program, National Oceanic and Atmospheric Administration, and the University of North Carolina Sea Grant Program under grants NA16RG2251 R/MRD-35 and NA16RG2251 R/MRD-49, and by the North Carolina Agricultural Research Service.

\section{Literature Cited}

Arendt, J. D. 1997. Adaptive intrinsic growth rates: an integration across taxa. Quarterly Review of Biology 72: 149-177.

Brodin, T., and F. Johansson. 2002. Effects of predatorinduced thinning and activity changes on life history in a damselfly. Oecologia 132:316-322.

Claessen, D., C. van Oss, A. M. De Roos, and L. Persson. 2002. The impact of size-dependent predation on population dynamics and individual life history. Ecology 83:1660-1675.

Crowder, L. B., R. A. Wright, K. A. Rose, T. H. Martin, and J. A. Rice. 1994. Direct and indirect effects of southern flounder predation on a spot population: experimental and model analyses. Pages 61-77 in D. J. Stouder, K. L. Fresh, and R. J. Feller, editors. Theory and application in fish feeding ecology. University of South Carolina Press, Columbia, South Carolina, USA.

Cushing, D. H. 1981. Fisheries biology: a study in population dynamics. University of Wisconsin Press, Madison, Wisconsin, USA.

De Roos, A. M., L. Persson, and E. McCauley. 2003. The influence of size-dependent life-history traits on the structure and dynamics of populations and communities. Ecology Letters 6:473-487.

Ebenman, B., and L. Persson. 1988. Dynamics of sizestructured populations - an overview. Pages 3-9 in B. Ebenman and L. Persson, editors. Size-structured populations: ecology and evolution. Springer-Verlag, Berlin, Germany.

Gerritsen, J., and J. R. Strickler. 1977. Encounter probabilities and community structure in zooplankton communities: a mathematical model. Journal of the Fisheries Research Board of Canada 34:73-82.

Harper, D. G., and R. W. Blake. 1988. Energetics of piscivorous predator-prey interactions. Journal of Theoretical Biology 134:59-76.

Hodson, R. G., J. O. Hackman, and C. R. Bennett. 1981. Food habits of young spots in nursery areas of the Cape Fear River Estuary, North Carolina. Transactions of the American Fisheries Society 110:495-501.

Lima, S. L. 1998. Stress and decision-making under the risk of predation: recent developments from behavioral, reproductive, and ecological perspectives. Advances in the Study of Behavior 27:215-290.

Metcalfe, N. B., and P. Monaghan. 2001. Compensation for a bad start: grow now, pay later? Trends in Ecology and Evolution 16:254-260.

Munch, S. B., and D. O. Conover. 2003. Rapid growth results in increased susceptibility to predation in Menidia menidia. Evolution 57:2119-2127. 
Olson, M. H. 1996. Predator-prey interactions in sizestructured fish communities: implications of prey growth. Oecologia 108:757-763.

Peacor, S. D. 2002. Positive effect of predators on prey growth rate through induced modifications of prey behaviour. Ecology Letters 5:77-85.

Peacor, S. D., and E. E. Werner. 2000. Predator effects on an assemblage of consumers through induced changes in consumer foraging behavior. Ecology 81:1998-2010.

Persson, L., J. Andersson, E. Wahlstrom, and P. Eklov. 1996. Size-specific interactions in lake systems: predator gape limitation and prey growth rate and mortality. Ecology 77: 900-911.

Persson, L., A. M. De Roos, D. Claessen, P. Bystrom, J. Lovgreen, S. Sjogren, R. Svanback, E. Wahlstrom, and E. Westman. 2003. Gigantic cannibals driving a whole-lake trophic cascade. Proceedings of the National Academy of Sciences 100:4035-4039.

Pope, J. G., J. G. Shepherd, and J. Webb. 1994. Successful surfriding on size-spectra: the secret of survival in the sea. Philosophical Transactions of the Royal Society B: Biological Sciences 343:41-49.

Preisser, E. L., D. I. Bolnick, and M. F. Benard. 2005. Scared to death? The effects of intimidation and consumption in predator-prey interactions. Ecology 86:501-509.

Relyea, R. A. 2002. The many faces of predation: how induction, selection, and thinning combine to alter prey phenotypes. Ecology 83:1953-1964.

Rice, J. A., L. B. Crowder, and E. A. Marschall. 1997. Predation on juvenile fishes: dynamic interactions between size-structured predators and prey. Pages $333-356$ in R. C. Chambers and E. A. Trippel, editors. Early life history and recruitment in fish populations. Chapman and Hall, London, UK.

Rice, J. A., L. B. Crowder, and K. A. Rose. 1993. Interactions between size-structured predator and prey populations: experimental test and model comparison. Transactions of the American Fisheries Society 122:481-491.

Scharf, F. S., J. A. Buckel, and F. Juanes. 2002. Size-dependent vulnerability of juvenile bay anchovy Anchoa mitchilli to bluefish predation: does large body size always provide a refuge? Marine Ecology Progress Series 233:241-252.

Sih, A., and B. Christensen. 2001. Optimal diet theory: when does it work, and when and why does it fail? Animal Behaviour 61:379-390.

Sinclair, A. F., D. P. Swain, and J. M. Hanson. 2002. Disentangling the effects of size-selective mortality, density, and temperature on length-at-age. Canadian Journal of Fisheries and Aquatic Sciences 59:372-382.

Sogard, S. M. 1997. Size-selective mortality in the juvenile stage of teleost fishes: a review. Bulletin of Marine Science $\mathbf{6 0}$ : $1129-1157$

Stoks, R., M. De Block, F. Van de Meutter, and F. Johansson. 2005. Predation costs of rapid growth: behavioural coupling and physiological decoupling. Journal of Animal Ecology $\mathbf{7 4}$ 708-715.

Van Buskirk, J., and K. L. Yurewicz. 1998. Effects of predators on prey growth rate: relative contributions of thinning and reduced activity. Oikos 82:20-28.

Watson, G. S. 1962. Goodness of fit tests on a circle II. Biometrika 48:57-63.

Werner, E. E., and J. F. Gilliam. 1984. The ontogenetic niche and species interactions in size-structured populations. Annual Review of Ecology and Systematics 15:393-425.

Werner, E. E., and S. D. Peacor. 2003. A review of traitmediated indirect interactions in ecological communities. Ecology 84:1083-1100.

Wilbur, H. M. 1988. Interactions between growing predators and growing prey. Pages $157-172$ in B. Ebenman and L. Persson, editors. Size-structured populations: ecology and evolution. Springer-Verlag, Berlin, Germany.

Williams, I. S. 1999. Slow-growth, high-mortality - a general hypothesis, or is it? Ecological Entomology 24:490-495.

\section{APPENDIX A}

Additional details of the pond experiment, including spot size, growth, and mortality and physical conditions of the ponds (Ecological Archives E087-144-A1).

\section{APPENDIX B}

Information on flounder growth in the pond experiment (Ecological Archives E087-144-A2).

\section{APPENDIX C}

Simulating the thinning effect of flounder predation on spot growth (Ecological Archives E087-144-A3).

\section{APPENDIX D}

Can size-dependent predation account for more of the observed shift in spot sizes? (Ecological Archives E087-144-A4). 